\title{
ICU Pad Project: application of modern computer technology in pediatric postoperative cardiac intensive care. Pilot study
}

\author{
Katarzyna Gendera ${ }^{1}$, Grzegorz Lipecki², Marcin Miedziński², Bartłomiej Prędki ${ }^{3}$, Wojciech Mrówczyński ${ }^{4}$ \\ ${ }^{1}$ Poznan University of Medical Sciences, Poland
}

${ }^{2}$ MA Study, Division of IT, University of Technology, Poznan, Poland

${ }^{3}$ Institute of IT, Division of IT, University of Technology, Poznan, Poland

${ }^{4}$ Department of Pediatric Cardiac Surgery, Poznan University of Medical Sciences, Poznan, Poland

Kardiochirurgia i Torakochirurgia Polska 2016; 13 (1): 83-89

After cardiac surgery procedures, all vital functions of the patient must be monitored carefully with particular focus on hemodynamic parameters. Optimizing the function of the circulatory system is the primary task of an intensive care physician as low cardiac output syndrome is one of the fundamental challenges of providing medical care to both children and adults after cardiac procedures. After a cardiac procedure, the cardiac output and, thereby, patient condition and prognosis, can be influenced by many factors, including arterial blood pressure, circulating fluid volume, arrhythmia as well as myocardial dysfunction resulting from open heart surgery using extracorporeal circulation [1, 2]. The evolution of hemodynamic parameters shortly after the procedure is usually difficult to predict. The situation may be additionally complicated by the presence of concomitant diseases or perioperative complications, specific properties of the procedure (palliative vs. total repair) and the heart defect, and the age of the patient (e.g., compensatory mechanisms are exhausted quicker in neonates and infants).
The described clinical scenario requires making correct therapeutic decisions that consider all the variables characterizing the patient's condition before the procedure (including, among others, the diagnosis) and in the recent past (e.g., during the procedure and the following hours) as well as variables registered on a current basis by systems designed to monitor vital parameters (both physical and biochemical). Information obtained from clinical examinations, nurse activities and observations, life support systems (mechanical ventilators, renal replacement therapy, mechanical circulatory support) as well as information associated with pharmacotherapy (e.g., the amount of fluids administered to the patient, dosage of pressor agents) must also be considered [3].

Making therapeutic decisions in the situation presented above involves analyzing significant amounts of data from various sources (Fig. 1). This makes tracking the changes of individual parameters on an ongoing basis particularly difficult. Moreover, the variety of information sources makes data analysis less effective due to potential time loss and

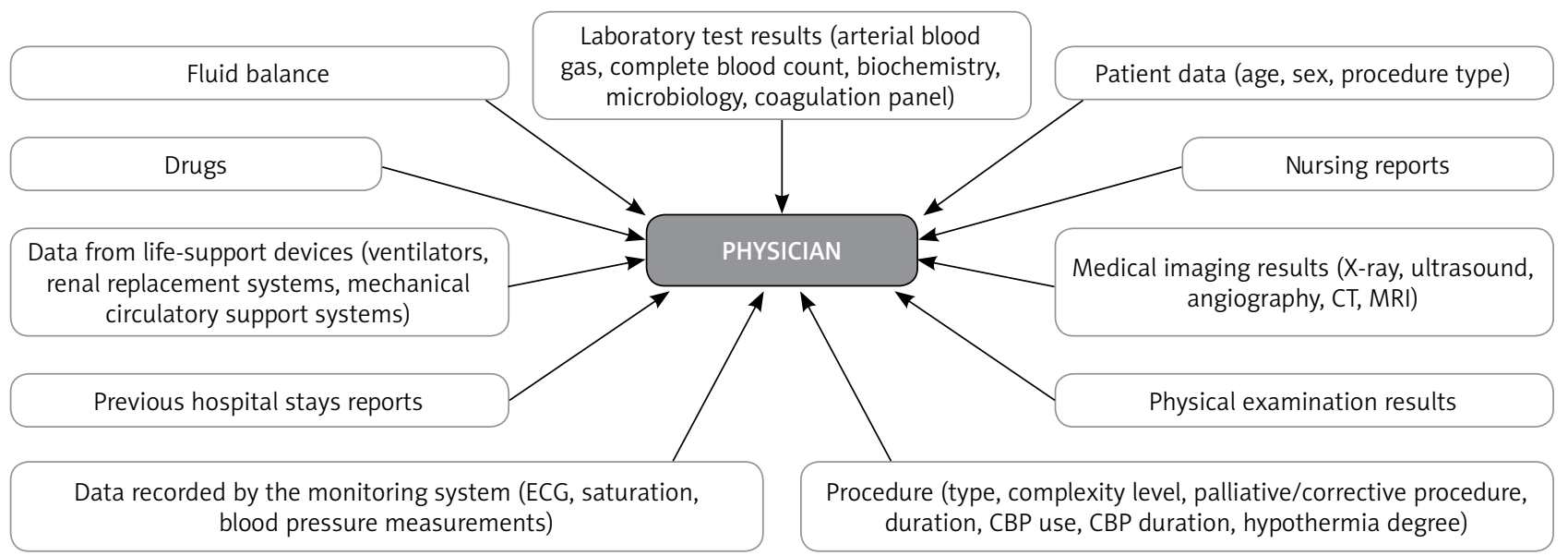

Fig. 1. Sources of information for physicians

Address for correspondence: Katarzyna Gendera, Poznan University of Medical Sciences, 64a Raczyńskiego St., 60-476 Poznań, Poland, phone: +48 509717844, e-mail: kasiagendera@wp.pl 
errors associated with the necessity of alternately gaining access to different systems. Additionally, the variety of information sources precludes uniform data presentation and introduction of mechanisms for detecting variables that fall outside of the norms set for a given patient [4].

Using a clinical system to integrate access to various data sources describing the patient's condition and enable clear analysis (on a current and retrospective basis) of all registered variables would surely facilitate the work of intensivists treating hemodynamically unstable patients after cardiac procedures. Such a system should be simple and intuitive to use, mobile, and easy to disinfect. An additional advantage comes with the ability to couple a visualization system with an expert system or a clinical decision support system (CDSS), which are able to analyze the incoming stream of medical data on a current basis, pinpointing pathological trends and associations between all variables from the whole period of hospitalization, thus aiding the intensive care physician $[5,6]$.

This paper describes a prototype of a system for integrating medical data, created on the basis of the foregoing rationale as part of the pilot program ICU Pad Project (IPP). The main component enabling communication between the user and the system is a mobile touch screen tablet. The system owes its development to intercollegiate cooperation with the Department of Information Technology of the Poznan University of Technology.

The ICU Pad Project was approved by the Bioethical Committee of the Poznań University of Medical Sciences (approval no. 837/12, date: September 13, 2012). Testing the system involved gathering sensitive information; for this reason, the system was isolated from the "external world" and the hospital medical network. Each time when the gathered data had to be exported outside of the hospital, sensitive data were anonymized. Work on the project involved the participation of physicians, nurses, as well as individuals who did not take part in the therapeutic process. Non-medical employees working on the project were required to sign confidentiality agreements and were pro-

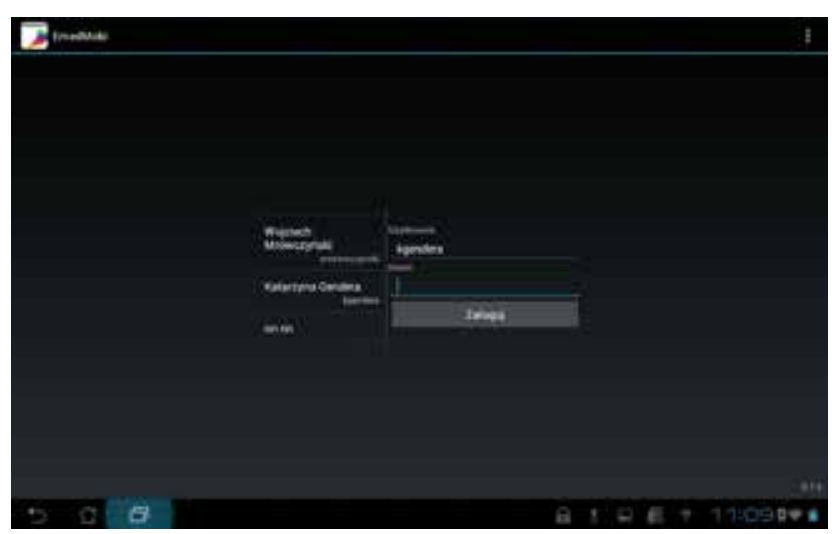

Fig. 2. Login screen. On the left side of the screen is the user list, on the right - the 'User' and 'Password' fields. After selecting the user, entering the password, and clicking the 'Log in' button, the user's credentials are verified, and the list of admitted patients is displayed on the screen hibited from disclosing the medical data they accessed. Encrypted wireless transmission between authorized devices only was used during system testing.

The system consists of a server based on the Windows Vista operating system with the following software installed: Postgress SQL database (v. 9.2, http://www.postgresql. org) for data storage, Apache Tomcat http server (v. 7.0.37, http://httpd.apache.org) for handling data transmission to the mobile component, and a "stationary" software component enabling the management of the system (admitting and discharging the patients, adding users, printing observation cards). The element serving the function of joining the server and mobile components was a WiFi router ensuring encrypted transmission and access control on the level of trusted MAC addresses (Media Access Control addresses).

The mobile component consisted in tablet devices (ASUS EeePAD Transformer, 32GB RAM, Android 4.1, https:// www.android.com).

In order to test the system, it was used to gather the data of 28 patients in stable clinical condition; during the same time, conventional medical documentation, standard for postoperative wards, was maintained.

\section{System operation}

After turning on the tablet, the user is presented with the login screen (Fig. 2).

After the user's credentials are verified, the system displays the list of patients on the postoperative ward, their birthdates, gender, and duration of hospitalization (Fig. 3).

Selecting one of the patient fields opens a window with the patient's card.

The heading of the patient card includes their first and last name, gender, PESEL number, and birthdate, as well as additional information: body mass, blood type, date of admission, and day of hospitalization. This part of the patient card remains unchanged: it is always visible on the screen after opening the patient card, regardless of which parameters are currently analyzed.

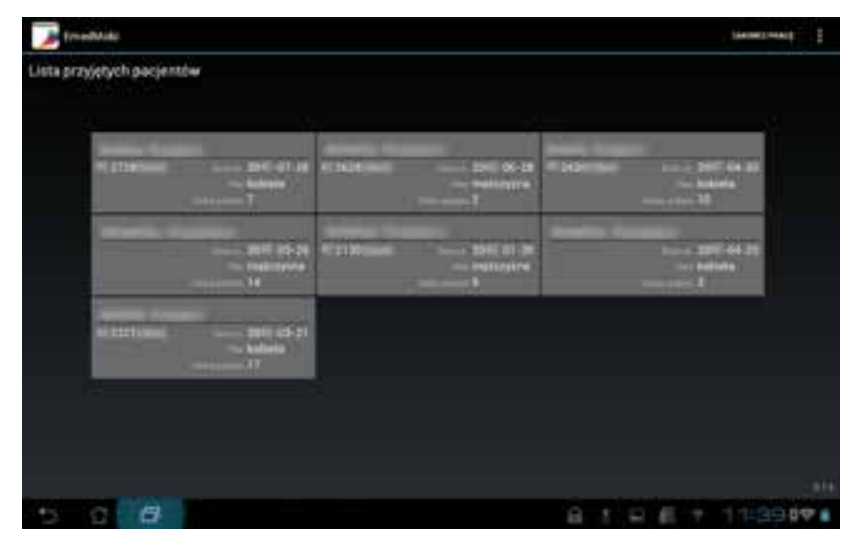

Fig. 3. The list of admitted patients. This view consists of rectangular tiles; each one is assigned to one patient. Each 'tile' contains basic information about the patient: first and last name, birthday, PESEL number, gender, and length of stay 
The information gathered on the patient card is grouped under tabs - the tabs available at the present stage of the system's development include: Hemodynamics, Fluid balance, Arterial Blood Gas, Complete Blood Count, Biochemistry, and Coagulation. Touching any of them allows the user to switch between the desired data sets.

The Hemodynamics tab (Fig. 4) presents data in the form of a chart. Each parameter is marked with a different color, which facilitates reading and interpreting the data. The chart may be moved in any direction, changing the time of origin of the presented data. Additionally, the chart's scale can be adjusted using touch gestures. The history of a selected parameter can also be displayed in a digital form.

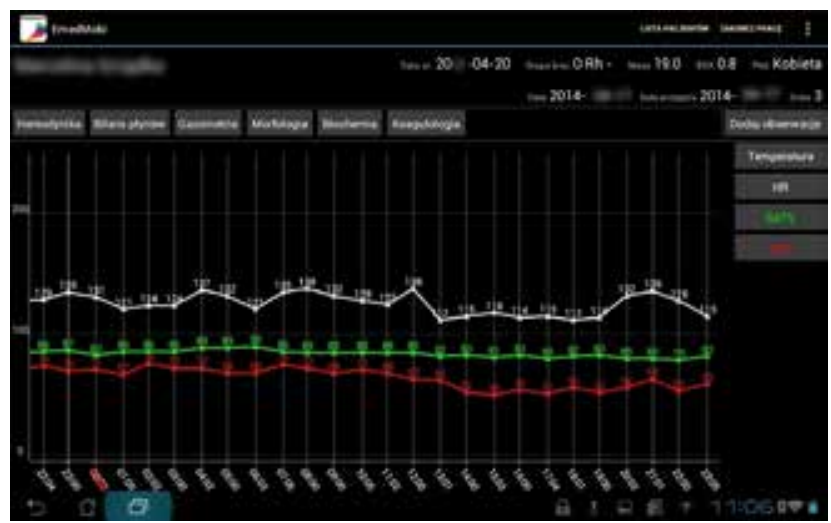

Fig. 4. Hemodynamics tab. This tab presents observations in the form of a chart. Each color is assigned to a specific parameter (e.g., white - heart rate, green - saturation, red - arteria blood pressure). On the right side of the screen, there are fields with names of parameters; selecting one of them enables the user to see the history of the parameter in a digital form. Above the parameter fields there is the 'Add observations' field; selecting it opens a window which enables adding observations to this tab

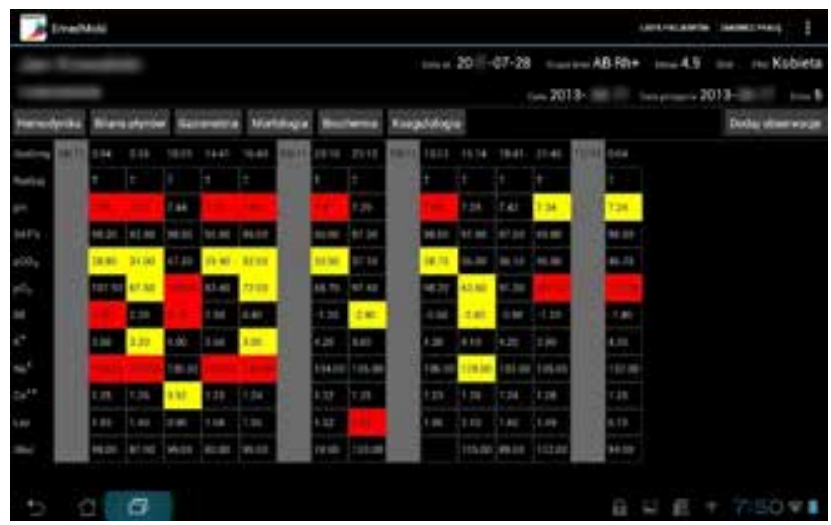

Fig. 5. Arterial Blood Gas tab. This tab presents measurement results in the form of a table. The first row of the table contains the hour at which the measurement was made. The results from individual days of the patient's stay are divided by columns with dates. Values below the norm are marked in yellow, while values above the norm are marked in red. In the right upper corner of the tab there is the 'Add observation' field which allows the user to add measurement results to the table
The Arterial Blood Gas, Biochemistry, Complete Blood Count, and Coagulation tabs present observations in the form of movable tables (Figs. 5 and 6).

The first row of the table contains the hour at which the measurement was made. Depending on the number of parameters in the rows, horizontal scrolling is enabled. Days on which the measurements were made are marked with a distinctive gray bar containing the date (day and month). The results table includes an automated alert mechanism, notifying the user when the norms for individual parameters are exceeded. Yellow is used to mark values below the norm, while red marks values above the norm. The norms are in accordance with the norms used in the hospital laboratory, defined for the current age and gender of the patient. This solution is meant to highlight parameter values of special note for the physician during therapy and to prevent potential mistakes when reading the results of laboratory examinations.

The FLUID BALANCE tab enables precise monitoring of the volume of fluids administered to and expelled by the patient during each day of hospitalization at the postoperative ward. When the data are entered into the system, the administered volume is divided into categories depending on the character of the fluid (colloid or crystalloid) and the method of administration (boluses, continous infusions, nasogastric tube). A similar division has been employed in order to determine the amount of fluids lost by a patient during each day (drainage volume divided into mediastinal drainage, left and right pleural drainage, volume of diuresis or hemodiafiltration, and volume of fluid contents evacuated through a gastric tube). The tab works in a manner similar to that of the BIOCHEMISTRY, COAGULATION, and COMPLETE BLOOD COUNT tabs - in the form of a table. Also, observations may be conveniently added and viewed in groups (Colloids, Drainage, Tube-supply, Tube-retention, Boluses, Continous infusions). The momentary value of the balance for the current day of hospitalization at the postoperative ward is calculated on a current basis for each group.

The individual groups in the Fluid Balance tab can be closed (Fig. 7) and opened (Fig. 8) by changing the selection of the 'Details' field available next to each group.

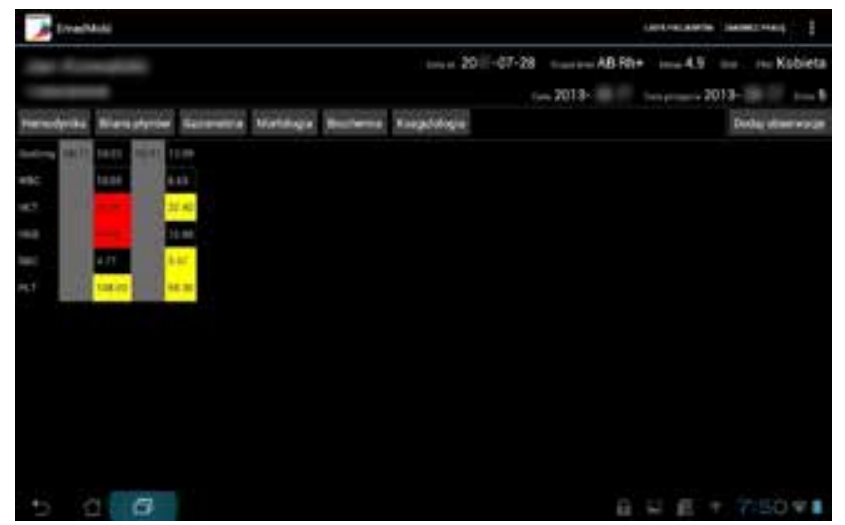

Fig. 6. Complete Blood Count tab. The data are presented in the form of a table; this tab also features an alert system notifying the user when the norms of specific values are exceeded 


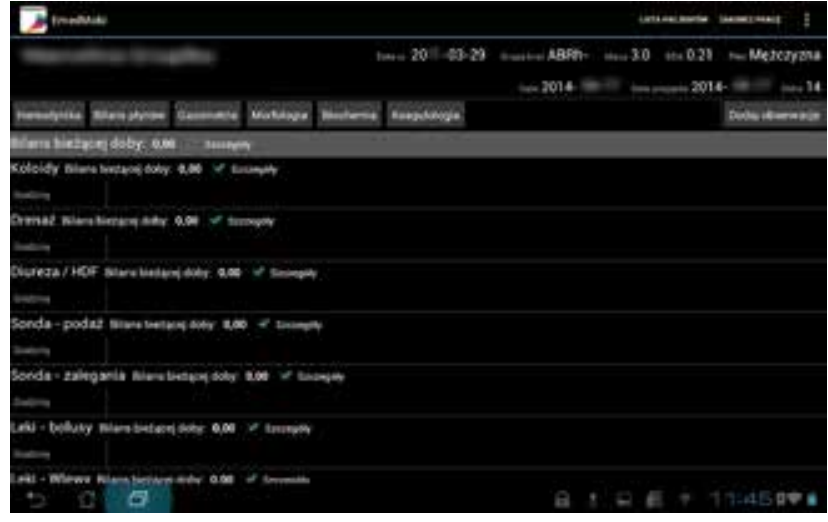

Fig. 7. Fluid balance tab - general view. The figure presents the Fluid Balance tab; in this mode, details concerning specific groups are hidden. At the top of the tab there is the fluid balance value for a given day. Below, specific categories are displayed, presenting the volumes administered to or expelled by the patient (from the top: Colloids, Drainage, Diuresis, Tube-supply, Tube-retention, Boluses, Continous infusions). Next to each category there is a 'Details' button enabling presentation of detailed information for each category as well as the balance for the current day for each category

In order to add an observation, the user must activate the tab to which they wish to add new information and push the 'Add observations' button. At this point, a window for adding observations will be displayed (Fig. 9). New data should be entered into the fields corresponding to the measured parameters; the data are saved using the 'Add observations' button. When entering the data, some of the fields can be left blank; this has no bearing on the saving of the remaining data. When the observations are successfully added, an appropriate message will be displayed, the adding window will be closed, and the tab will be refreshed. The default registered hour and date of an added observation is the time of entry, but this can be changed for each observation. The frequency of entering the data is at the user's discretion.

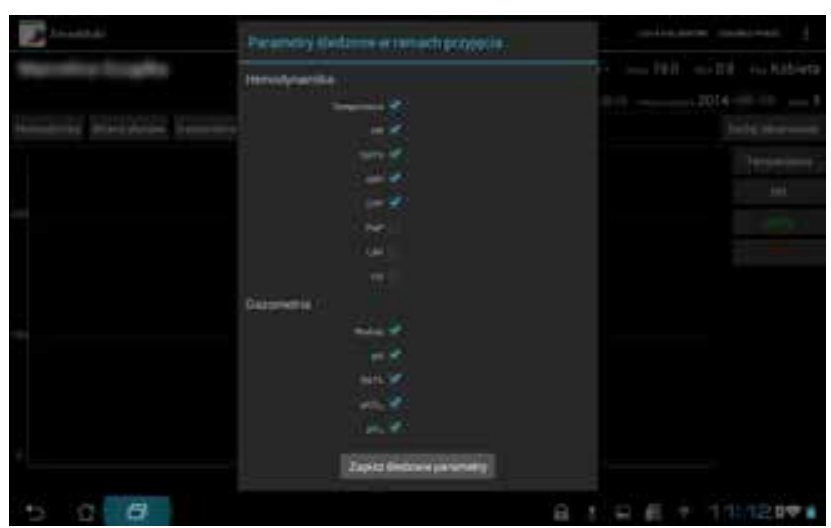

Fig. 9. Adding observations. Each tab features an 'Add observations' option. After clicking the button, the system displays a list of parameters for which values can be added. The top of the window features fields in which the day and hour of the observation is automatically entered. After the added observations are confirmed, the results are saved by the system

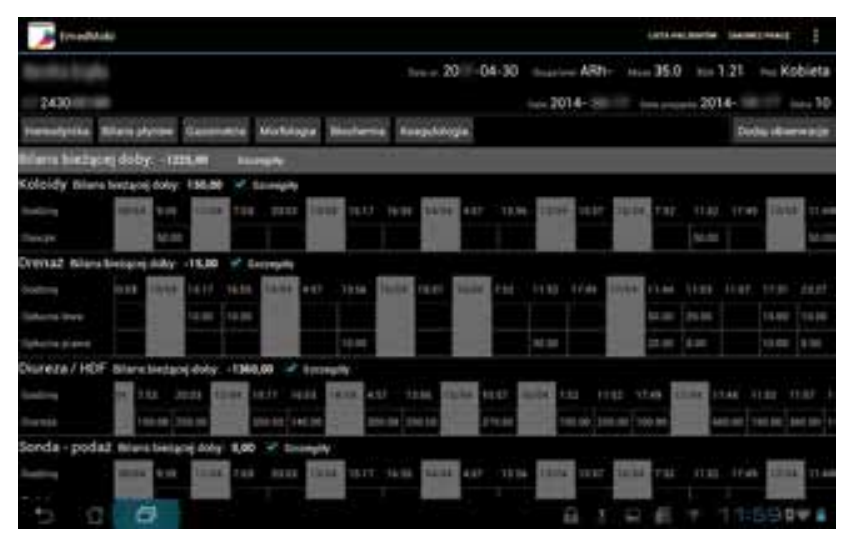

Fig. 8. Fluid balance tab - detailed view. The figure presents the tab showing details for each parameter group. Additional information includes the hour of the measurement and the amount administered to or expelled by the patient. Clicking for more details allows the user to display not only volume changes noted for the current day, but also the values noted on previous days, separated by columns containing the dates on which the information was entered

The IPP system enables the user to select parameters of interest only; viewing all available data is not necessary. The window presenting all parameters available in the system is divided in accordance with the names of the tabs relevant for the observation types (Fig. 10). Only the selected parameters are available in the window for adding observations. This solution enables the physician to focus on the parameters that are especially important to be monitored for a given patient.

After logging into the system, the user may end the session at any time. After ending the session, the current user will be logged out, and the login screen will be displayed.

The system has undergone its first tests conducted in clinical conditions. The testing focused primarily on processing the hemodynamic variables and the results of selected laboratory examinations. The test was conducted

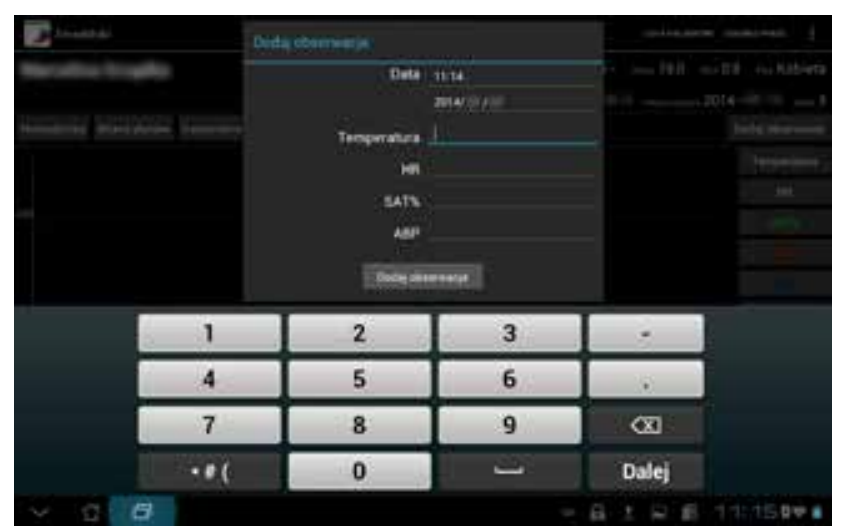

Fig. 10. Selecting observed parameters. The system enables selection of observed parameters. The 'Parameters monitored on admission' window contains a list of the parameters and shows to which tab they belong. The user can select the parameters that will be added and monitored for a given patient. The observed parameters can be changed at any time 
by 6 nurses during fourteen 12-hour shifts. The patients included in the test had been operated on due to the following types of heart defects: ASD II (atrial septal defect type 2), AS (aortic stenosis), DORV (double outlet right ventricle), VSD (ventricular septal defect), ToF (tetralogy of Fallot), PA (pulmonary atresia), BWG (Bland-White-Garland syndrome), AVSD (atrioventricular septal defect), CoA (coarctation of the aorta), d-TGA (transposition of the great arteries), ASD sinus venosus (atrial septal defect sinus venosus), ASD I (atrial septal defect type 1). The following parameters were registered: body temperature, heart rate, oxygen saturation, arterial blood pressure, pulmonary artery pressure, cardiac output, central venous pressure (Fig. 4), arterial blood gas ( $\mathrm{pH}$, blood saturation, partial pressure of carbon dioxide, partial pressure of oxygen, base excess, concentrations of potassium, sodium, and calcium ions, lactate concentration, and glucose concentration) (Fig. 5), coagulation parameters (fibrinogen concentration, prothrombin ratio, INR, prothrombin time, partial thromboplastin time, D-dimer concentration, antithrombin III concentration), complete blood count (white blood cell count, red blood cell count, and blood platelet count in blood volume, as well as hematocrit and hemoglobin concentration in blood) (Fig. 6). Information concerning the fluid balance was also recorded (measurement of the volume drained from the right and left pleura, diuresis value and/or the volume expelled through hemodiafiltration, the volume of contents evacuated from the gastric tube, the volume of administered colloid solutions, the volume of fluids administered through the gastric tube, the volume of fluids provided to the patients through the administration of agents in boluses and infusions) (Figs. 7 and 8).

Before receiving the tablets, the nurses received approx. 30 minutes of hands-on training and were able to practice all possible scenarios of entering and accessing data.

The IPP tablet system was well received by the medical personnel. The ease and intuitiveness of the touch interface enabled its uncomplicated use as a tool for gathering and visualizing hemodynamic data and results of biochemical analyses in selected patients. At the same time, suggestions for improvements, as well as information about glitches, were recorded.

\section{Discussion}

The prototype of the IPP system presented above appears to be one of the first attempts to employ mobile touchsensitive devices in the postoperative care provided to children after congenital heart defect procedures. The impulse behind the creation of the system was the need to improve the conventional system of documenting the postoperative course which has been on paper observation cards. There are many unquestionable drawbacks of the traditional system: the data can be incomplete; the paper cards can be easily lost; their size is cumbersome; mistakes can be made when the results are copied (there are no mechanisms of error control); the cards are unhygienic and can be a habitat for hospital microorganisms; it is difficult to process the gathered data; using the cards consumes paper, and archiving is cumbersome.

The electronic IPP system eliminates all of the abovementioned problems:

- the data can be gathered and integrated using one medium,

- the relationships between many variables can be visualized in a straightforward manner,

- the data can be accessed quickly,

- the system automatically visualizes the parameters whose norms are exceeded,

- the data can be analyzed on a current basis using the tablet by one or multiple individuals (video access, potentially with an option of teleconsultation),

- entering data is expedited, and the system checks for errors when the data are entered,

- the data are stored on the server in real time (data security),

- the system saves space (no paper documentation),

- the tablets are more hygienic.

Other advantages include the fact that the system can be integrated with existing information systems and treatment quality control mechanisms can be introduced.

The Intensive Care Unit of the Nicosia General Hospital has employed software similar to IPP (but without the use of tablets) called "Intensive Care Window" (ICW) [7]. The system consists in an ICW application installed on standard computers and a bedside controller collecting data from the cardiac monitor, respirator, and gasometric measurement equipment. The ICW application enables real-time presentation of the gathered data in a text and graphic form. Its users can highlight and mark variable changes of interest and monitor the clinical parameters us ing alerts that notify the user when any unfavorable changes occur. The software also enables offline data analysis. This function may be used during normal system operation and does not interfere with the process of gathering and storing data. Each piece of information gathered from a patient is identified (unambiguously assigned to a given patient) and immediately entered into the database. Helpfully, the application includes an efficient search system allowing the user to search for specific information in the gathered data [7]. Similarly, the presented IPP systems enables the graphic and numeric presentation of results, their retrospective analysis, and quick access to each moment of postoperative observation. Multiple individuals can access the data remotely, potentially from any hospital location with network access.

One of the primary goals of introducing the IPP system is to integrate and visualize medical data originating from different sources. This concerns mostly parameters registered by cardiac monitors and respirators, results of laboratory investigations, as well as information obtained during examinations performed by nurses and physicians. Integrating and visualizing the aforementioned data using one mobile medium undoubtedly allows intensive care physicians to better perceive the stream of information they are faced with when providing care to hemodynamically unstable patients. Apart from this aspect, integrating medi- 
cal data has other advantages as the information gathered and made available by the system can include information obtained on admission, results of preoperative diagnostic examinations, as well as descriptions of the procedure, the postoperative course, and the patient's condition on discharge. The ability to share information concerning the postoperative course in an orderly fashion with medical personnel from other wards when the patient is discharged from the postoperative ward for further treatment appears to be especially advantageous [8, 9].

Apart from the practical benefits, employing the IPP system offers the following scientific advantages:

- in terms of information technology: development of new user-friendly interfaces for entering data along with mechanisms for teleconsultation as well as visualization and analysis of medical data generated by the touch screen tablet,

- in terms of medical sciences: development of clinical decision support systems [6, 10],

- intelligent system alerts (elimination of false alarms) [11],

- the ability to analyze the postoperative course of selected heart defects with consideration to previously unstudied parameters or to identify new risk factors for adverse events,

- an opportunity to optimize postoperative treatment of selected heart defects based on the analysis of the data gathered by the system [12].

Systems that integrate medical data, such as the presented IPP or the aforementioned ICW systems, are able to generate large quantities of data, especially if the registered parameters include direct recordings of hemodynamic curves and ECG evolution, registered automatically, or the results of imaging examinations. As the cost of storing such information is no longer a limitation, considering the current technological capabilities, creating high definition medical databases describing the condition of patients undergoing intensive medical care is becoming a reality. This is one of important aspects of implementing and using IPP, ICW, and other such systems [13, 7].

A flagship example of a high definition medical database is the MIMIC-II project (Multiparameter Intelligent Monitoring in Intensive Care II) [13-15]. Apart from its practical advantages, MIMIC-II can be employed in various scientific endeavors, owing to the fact that all the information in its database can be shared after the removal of sensitive data (anonymization) [16]. The literature features reports in which data from the MIMIC-II project were analyzed offline. The published studies regarded, e.g., determining ARDS risk in patients undergoing mechanical ventilation longer than 48 hours [17], hypertension as a risk factor for acute kidney injury [18], the estimation of cardiac output values based on the arterial blood pressure curve [19], the identification of mortality risk factors [20], the possibility of discontinuing pressor agents within the first 12 hours after cardiac surgery procedures [20], and the assessment of factors affecting the development of septic shock [20]. These examples demonstrate the enormous scientific potential, enabling the generation of new knowledge, which stems from the ability to analyze data whose storage and assessment would not be possible without systems designed for the integration of medical information.

The experiences accumulated during the design and creation of the IPP system as well as literature reports point to the significant number of challenges that must be overcome in order to create an efficient high definition medical database. One of the main challenges is the fact that the data, originating from various sources, must be properly attributed to a given patient, e.g., by using a marking system based on personal data [13]. At the current stage of IPP development, the automation of this process has been unnecessary as these tasks are performed by tablet operators.

Apart from offline data mining, the information gathered by a medical data integration system can also be used to create expert systems and clinical decision support systems $[5,6])$. Such a system could recognize certain problems typical for intensive treatment after cardiac surgery procedures (e.g., hypotension, hypovolemia, vasodilatation, or SIRS) and introduce appropriate treatment. Studies have shown that in each clinical scenario, the tested clinical decision support system was able to select therapeutic procedures similar to those suggested by intensive care physicians. As a result, the system was able to control hemodynamic parameters and maintain their values within the norm $[5,6]$.

\section{Limitations}

The presented prototype of the IPP system constitutes, in its current form, evidence that the concept and premises assumed during its design are correct: a proof of concept. The fact that the nurses received the product well and are eager to use it and to contribute to its development stimulates its authors to further efforts. More work is required to tackle software glitches on an ongoing basis and eliminate the limitations related to the system's current isolation from the hospital network. As a pilot system, the IPP project was designed in isolation from the existing hospital network and the external world (intranet/ internet) in order to protect patient data and avoid potential conflicts with the current IT systems during these early stages of IPP development. This isolation precludes the automatic gathering of data (e.g., laboratory results) from the hospital system or autonomous data generating equipment (e.g., cardiac monitors, respirators). Although integration with the hospital IT system based on open communication protocols ( $\mathrm{HL}-7)$ is planned, transferring data from the aforementioned autonomous equipment may pose a significant challenge due to the large number of restricted data exchange protocols [7]. The system would also be significantly improved if it was provided with capabilities to integrate information concerning drug interactions, suggested dosage dependent on age, gender, and body mass, as well as the patient's condition and concomitant diseases. 


\section{Perspectives}

The development of the IPP system may improve the comfort and safety of work in intensive care units and contribute to the improvement of treatment outcomes in patients after congenital heart defect procedures. At the same time, IPP can be a unique tool for gathering data that will be useful in further clinical studies as well as a platform for the development of medical expert systems.

\section{Disclosure}

Authors report no conflict of interest.

\section{References}

1. Currey J, Botti M. The hemodynamic status of cardiac surgical patients in the initial 2-hrecovery period. Eur J Cardiovasc Nurs 2005; 4: 207-214.

2. Foot CL, Frazer JF, Mullany DV. Common complications after cardiacsurgery in adults. Current Anesthesia \& Critical Care 2005; 16: 331-345.

3. Data integration in cardiac surgery health care institution: Experience at G. Pasquinucci Heart Hospital. Computers in Cardiology 2008; 2008: 287-290.

4. Heldt T, Verghese GC. Model-based data integration in clinical environments. Conf Proc IEEE Eng Med Biol Soc 2010; 2010: 5209-5212.

5. Denaï MA, Mahfouf M, Ross JJ. A hybrid hierarchical decision support system for cardiac surgical intensive care patients. Part I: Physiological modelling and decision support system design. Artif Intell Med 2009; 45: 35-52.

6. Ross JJ, Denaï MA, Mahfouf M. A hybrid hierarchical decision support system for cardiac surgical intensive care patients. Part II. Clinical implementation and evaluation. Artif Intell Med 2009; 45: 53-62.

7. Stylianides N, Dikaiakos MD, Gjermundrød H, Panayi G, Kyprianou T. Intensive care window: real-time monitoring and analysis in the intensive care environment. IEEE Trans Inf Technol Biomed 2011; 15: 26-32.

8. Taddei A, Dalmiani S, Vellani A, Rocca E, Piccini G, Carducci T, Gori A, Borghini R, Marcheschi P, Mazzarisi A, Salvatori C, Macerata A. Data integration in cardiac surgery health care institution: Experience at G. Pasquinucci Heart Hospital. Computers in Cardiology 2008; 35: 287-290.
9. Jordan D, Rose SE. Multimedia abstract generation of intensive care data: the automation of clinical processes through Al methodologies. World J Surg 2010; 34: 637-645.

10. Adlassing K. The Section on Medical Expert and Knowledge-Based Systems at the Department of Medical Computer Sciences of the University of Vienna Medical School. Artif Intell Med 2001; 21: 139-146.

11. Aboukhalil A, Nielsen L, Saeed M, Mark RG, Clifford GD. Reducing false alarm rates for critical arrhythmias using the arterial blood pressure waveform. J Biomed Inform 2008; 41: 442-451.

12. Hekmat K, Doerr F, Kroener A, Heldwein M, Bossert T, Badreldin AMA, Lichtenberg A. Prediction of mortality in intensive care unit cardiac surgery patients. Eur J Cardiothorac Surg 2010; 38: 104-109.

13. Saeed M, Villarroel M, Reisner AT, Clifford G, Lehman L-W, Moody G, Heldt T, Kyaw TH, Moody B, Mark RG. Multiparameter Intelligent Monitoring in Intensive Care II: a public-access intensive care unit database. Crit Care Med 2011; 39: 952-960.

14. Lee J, Scott DJ, Villarroel M, Clifford GD, Saeed M, Mark RG. Open-access MIMIC-II database for intensive care research. Conf Proc IEEE Eng Med Biol Soc 2011; 2011: 8315-8318.

15. Saeed M, Lieu C, Raber G, Mark RG. MIMIC II: a massive temporal ICU patient database to support research in intelligent patient monitoring. Comput Cardiol 2002; 29: 641-644.

16. Douglass M, Clifford GD, Reisner A, Moody GB, Mark RG. Computer-assisted de-identification of the free text in the MIMIC II database. Computers in Cardiology 2004; 31: 341-344.

17. Jia X, Malhotra A, Saeed M, Mark RG, Talmor D. Risk factors for ARDS in patients receiving mechanical ventilation for $>48 \mathrm{~h}$. Chest 2008; 133: 853-861.

18. Lehman L, Saeed M, Moody G, Mark R. Hypertension as a risk factor for acute kidney injury in ICU patients. Computing in Cardiology 2010; 37: 1095-1098.

19. Sun JX, Reisner AT, Saeed M, Mark RG. Estimating cardiac output from arterial blood pressure waveforms: a critical evaluation using the MIMIC II database. Computers in Cardiology 2005; 32: 295-298.

20. Hug CW. Predicting the risk and trajectory of intensive care patients using survival models. PhD thesis, Massachusetts Institute of Technology, Department of Electrical Engineering and Computer Science, 2009. 